\title{
BRIEF COMMUNICATION \\ Epigenetic analysis confirms no accelerated brain aging in schizophrenia
}

\author{
Joanne Voisey ${ }^{1}$, Bruce R. Lawford ${ }^{2}$, C. Phillip Morris (iD), Leesa F. Wockner ${ }^{3}$, Ernest P. Noble ${ }^{4}$, Ross McD Young ${ }^{5}$ and Divya Mehta ${ }^{2}$
}

Epigenetic aging is associated with several biological mechanisms and diseases. We assessed two brain data sets, one small ( $n=48)$ and one large $(n=392)$, to test epigenetic aging in schizophrenia. DNA methylation age from frontal cortex was significantly correlated with chronological age but no significant differences in DNA methylation age acceleration between schizophrenia cases and controls were observed in both data sets. Our results were consistent with a previous study investigating schizophrenia and epigenetic aging in superior temporal gyrus. Future studies targeting different brain regions and defined cell types are warranted to further investigate accelerated brain aging in schizophrenia.

npj Schizophrenia (2017)3:26; doi:10.1038/s41537-017-0026-4

\section{INTRODUCTION}

Several lines of evidence have pointed towards the hypothesis of accelerated brain aging in schizophrenia. ${ }^{1,2}$ In recent years, DNA methylation of $353 \mathrm{CpG}$ sites (epigenetic clock) have been used to accurately estimate the biological age of human tissues and cell types. ${ }^{3}$ Studies have found epigenetic aging to be associated with Alzheimer's disease, ${ }^{4}$ obesity ${ }^{5}$ and cancer. ${ }^{6}$ Only one study has investigated epigenetic aging in schizophrenia. ${ }^{7}$ Using postmortem brain samples from the superior temporal gyrus $(n=44)$, no acceleration of brain aging in schizophrenia was identified.

The aim of this study was to test the hypothesis of accelerated aging in schizophrenia by assessing epigenetic age in brain tissue of individuals with schizophrenia. Using a different brain region, the frontal cortex, we investigated a total of 440 samples including data from our previous study ${ }^{8}(n=48)$ as well as an independent published data ${ }^{9}(n=392)$.

\section{METHODS}

Genome-wide DNA methylation analysis was generated from post-mortem human brain tissue (frontal cortex) from 24 individuals with schizophrenia and 24 unaffected controls. DNA methylation was assessed using the Illumina Infinium HumanMethylation450 Bead Chip, details are described in a previous study. ${ }^{8}$ DNA methylation-based age prediction was performed using the statistical pipeline developed by Horvath. ${ }^{3}$ The raw data were normalized using BMIQ normalization method. To measure epigenetic age acceleration effects, we regressed DNAm age on chronological age to obtain the DNAm age acceleration residuals. Hence, age acceleration would denote individuals who appear to be older than their chronological age. Next, we regressed DNAm age acceleration residuals against the group status, adjusting for gender, brain post-mortem interval and cause of death. Similar methods were used for the independent published replication data set as in the original study adjusting for gender, four principal components and ethnicity. ${ }^{9}$ From the published data set, we limited our analysis to adult samples that passed the QC including 217 controls and 175 Schizophrenia, comprising a total of 392 samples (Supplementary Table 1).
Ethics approval for the project was obtained from the Human Research Ethics Committee of the Queensland University of Technology.

Data availability

Discovery data set https://www.ncbi.nlm.nih.gov/geo/query/acc.cgi? acc=GSE61107

Replication data set https://www.ncbi.nlm.nih.gov/geo/query/acc.cgi? acc=GSE74193

\section{RESULTS}

Demographics of the samples are shown in Table 1. There was a significant difference in PMI and age across the samples, but no significant differences in DNA methylation age acceleration. All results are indicated in Fig. 1. In the overall sample $(n=48)$, DNA methylation age was significantly correlated with chronological age $(r=0.92, p$-value $=4.626603 \mathrm{e}-20)$. Similar results were observed in schizophrenia cases $(r=0.92$ and $p$-value $=8.62 \mathrm{e}$ $10)$ and in controls ( $r=0.82$ and $p$-value $=7.2 \mathrm{e}-07)$.

When comparing the groups, no significant differences in DNAm age acceleration was seen between the schizophrenia cases and controls ( $p$ value $=0.08$ ), after adjusting for covariates. No differences in DNAm age acceleration were observed when stratifying by gender or by taking the mean or median age of the sample ( $p$-value $>0.05$ ). The average delta age (DNAm-age-chronological age) in the schizophrenia cases was -10.4 and that in the controls was -14.8 years, indicating that on average, the controls had 4 years increased age acceleration than the schizophrenia cases.

We replicated our findings using 392 samples (217 controls and 175 Schizophrenia) from another study which investigated DNA methylation in prefrontal cortex. ${ }^{9}$ In the overall replication sample, DNA methylation age was significantly correlated with chronological age $(r=0.9454137, p$-value $=5.237090 \mathrm{e}-192)$. Similar results were observed in schizophrenia cases $(r=$ $0.9292716, p$-value $=9.775933 \mathrm{e}-77)$ and in controls $(r=0.9517476, p$-value $=2.853058 \mathrm{e}-112$ ). As in the discovery sample, no significant differences in DNAm age acceleration was seen between the schizophrenia cases and controls ( $p$-value $=0.916$ ) also when stratifying by gender or by taking the mean or median age of the sample ( $p$-value $>0.05)$. The average delta age

\footnotetext{
${ }^{1}$ School of Biomedical Sciences, Faculty of Health, Institute of Health and Biomedical Innovation, Queensland University of Technology, Brisbane, QLD, Australia; ${ }^{2}$ School of Psychology and Counselling, Faculty of Health, Institute of Health and Biomedical Innovation, Queensland University of Technology, Brisbane, QLD, Australia; ${ }^{3}$ Queensland Institute of Medical Research, Brisbane, QLD, Australia; ${ }^{4}$ Department of Psychiatry and Biobehavioral Sciences, University of California, Los Angeles, CA, USA and ${ }^{5}$ Faculty of Health, Institute of Health and Biomedical Innovation, Queensland University of Technology, Brisbane, QLD, Australia Correspondence: Divya Mehta (divya.mehta@qut.edu.au)
}

Received: 11 May 2017 Revised: 9 July 2017 Accepted: 17 July 2017 Published online: 04 September 2017 
Table 1. Demographics of the 48 samples used in the study

\begin{tabular}{lllll}
\hline & $\begin{array}{l}\text { Total }(n=48) \\
\text { Mean [SE] or } N[\%]\end{array}$ & Scz $(n=24)$ & Controls $(n=24)$ \\
\hline Age & $61.66[2.80]$ & $52[4.5]$ & $71.3[2.0]$ & 0.000192 \\
PMI & $18.84[1.32]$ & $24[2.17]$ & $14.1[0.67]$ & $19[79 \%]$ \\
Gender-males [\%] & $35[73 \%]$ & $16[67 \%]$ & 0.000075 \\
Cause of dealth-suicide & $5[10 \%]$ & $5[21 \%]$ & $-1.35[1.17]$ & 0.451 \\
Age acceleration residual & $1.33 \mathrm{e}-15[0.80]$ & $1.30[0.93]$ & 0.018 \\
\hline
\end{tabular}

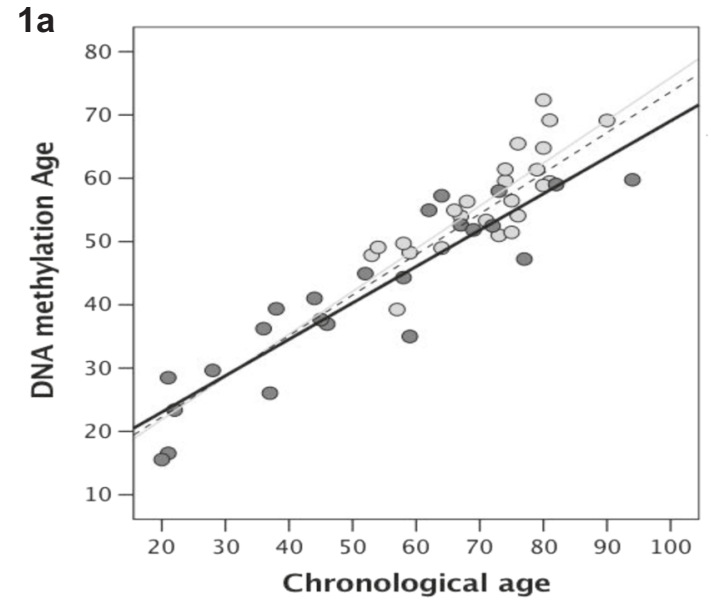

1c

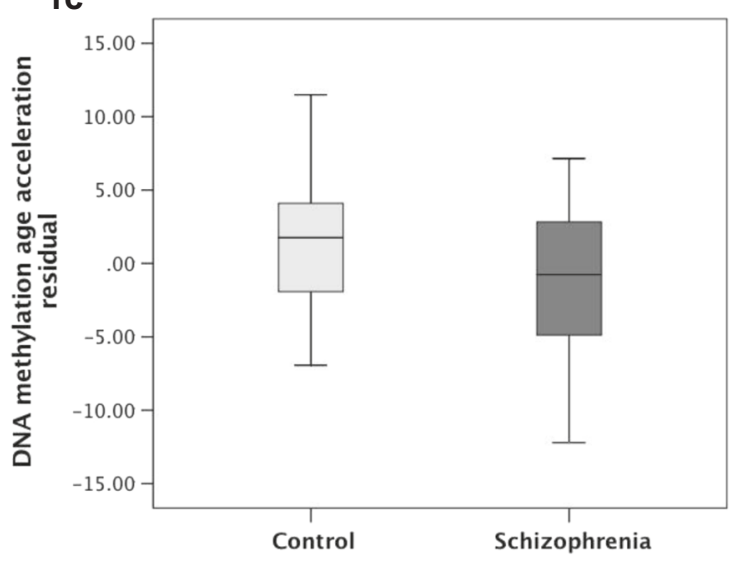

1b

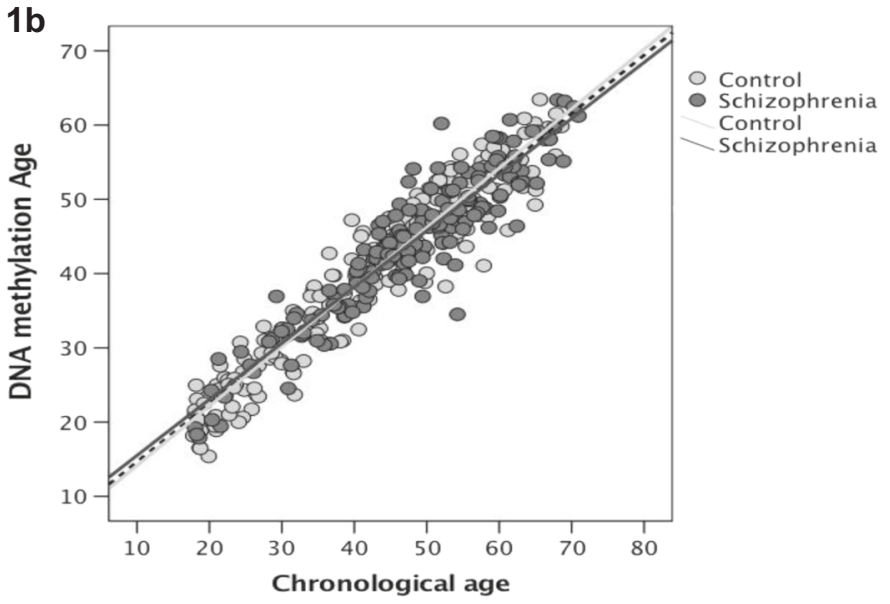

$1 d$

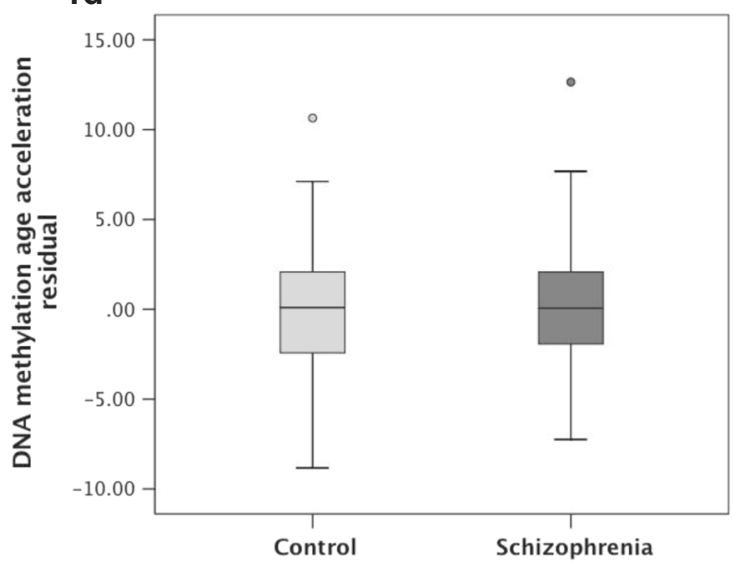

Fig. 1 No evidence of accelerated aging in brain tissue in schizophrenia- $\mathbf{a}$ and $\mathbf{b}$ indicate the significant correlation between the chronological and epigenetic age in our sample (a) $(r=0.92, p$-value $=4.626603 \mathrm{e}-20)$ and the replication sample $(\mathbf{b})(r=0.9454137 p$-value 5.237090e-192). $\mathbf{c}$ and $\mathbf{d}$ indicate no differences in DNA methylation age acceleration in our sample $\mathbf{c}(p$-value $=0.095)$ and the replication sample (d) $(p$-value $=0.702)$

(DNAm-age-chronological age) in the in the controls was -2.3 years and that schizophrenia cases was -3.7 .

\section{CONCLUSIONS}

This is the first study in frontal cortex and only the second study using brain tissue to investigate the relationship between epigenetic aging and schizophrenia. Although schizophrenia is associated with age-related physiological factors as well as significant decrease in average life span, ${ }^{10}$ we were unable to confirm accelerated aging in our study. These results suggest that brain volume loss observed in schizophrenia might be explained by pathological processes other than accelerated aging. Our results are limited since this is a cross sectional study and we were unable to assess progressive age acceleration that might occur in the brain of individuals with schizophrenia. Also, it is likely that there might be other confounding factors that might influence our results.
In conclusion, our findings in frontal cortex of individuals with schizophrenia are consistent with those from McKinney et al. ${ }^{7}$ who reported lack of evidence for accelerated epigenetic aging in schizophrenia superior temporal gyrus region of the brain. Nevertheless, we cannot rule out the possibility of other aging mechanisms that might be independent of epigenetic aging in schizophrenia brain and/or accelerated epigenetic aging that might be present in other tissues. Future studies aimed at testing the accelerated aging hypothesis in other brain regions as well as defined cell types will further uncover the hypothesis of accelerated brain aging in schizophrenia.

\section{ACKNOWLEDGEMENTS}

This work was financially supported by the Nicol Foundation and the Institute of Health and Biomedical Innovation, QUT. 


\section{AUTHOR CONTRIBUTIONS}

J.V. substantially contributed to the study design, writing and critical editing of the manuscript. B.L., C.P.M., L.W., E.N. and R.M.Y. substantially contributed to the study design and critical editing of the manuscript. D.M. substantially contributed to the study design, statistical analyses, writing and critical editing of the manuscript. All authors reviewed and approved the final version of the manuscript for publication.

\section{ADDITIONAL INFORMATION}

Supplementary Information accompanies the paper on the npj Schizophrenia website (doi:10.1038/s41537-017-0026-4).

Competing interests: The authors declare that they have no competing financial interests.

Publisher's note: Springer Nature remains neutral with regard to jurisdictional claims in published maps and institutional affiliations.

\section{REFERENCES}

1. Koutsouleris, N. et al. Accelerated brain aging in schizophrenia and beyond: a neuroanatomical marker of psychiatric disorders. Schizophr. Bull. 40, 1140-1153 (2014).

2. Schnack, H. G. et al. Accelerated brain aging in schizophrenia: a longitudinal pattern recognition study. Am. J. Psychiatry 173, 607-616 (2016).

3. Horvath, S. DNA methylation age of human tissues and cell types. Genome Biol. 14, R115 (2013).

4. Levine, M. E., Lu, A. T., Bennett, D. A. \& Horvath, S. Epigenetic age of the prefrontal cortex is associated with neuritic plaques, amyloid load, and Alzheimer's disease related cognitive functioning. Aging 7, 1198-1211 (2015).
5. Horvath, S. et al. Obesity accelerates epigenetic aging of human liver. Proc Natl Acad Sci USA 111, 15538-15543 (2014).

6. Ambatipudi, S. et al. DNA methylome analysis identifies accelerated epigenetic ageing associated with postmenopausal breast cancer susceptibility. Eur. J. Cancer 75, 299-307 (2017).

7. McKinney, B. C., Lin, H., Ding, J., Lewis, D. A., \& Sweet, R. A. DNA methylation evidence against the accelerated aging hypothesis of schizophrenia. NPJ Schizophr. doi:10.1038/s41537-41017-40017-41535 (2017).

8. Wockner, L. F. et al. Genome-wide DNA methylation analysis of human brain tissue from schizophrenia patients. Transl. Psychiatry 4, e339 (2014).

9. Jaffe, A. E. et al. Mapping DNA methylation across development, genotype and schizophrenia in the human frontal cortex. Nat. Neurosci. 19, 40-47 (2016).

10. Kirkpatrick, B., Messias, E., Harvey, P. D., Fernandez-Egea, E. \& Bowie, C. R. Is schizophrenia a syndrome of accelerated aging?. Schizophr. Bull. 34, 1024-1032 (2008).

Open Access This article is licensed under a Creative Commons Attribution 4.0 International License, which permits use, sharing, adaptation, distribution and reproduction in any medium or format, as long as you give appropriate credit to the original author(s) and the source, provide a link to the Creative Commons license, and indicate if changes were made. The images or other third party material in this article are included in the article's Creative Commons license, unless indicated otherwise in a credit line to the material. If material is not included in the article's Creative Commons license and your intended use is not permitted by statutory regulation or exceeds the permitted use, you will need to obtain permission directly from the copyright holder. To view a copy of this license, visit http://creativecommons. org/licenses/by/4.0/.

(C) The Author(s) 2017 